\section{REDIMAT}

Journal of Research in Mathematics Education
Hipatia Press

www.hipatiapress.com

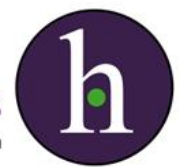

Instructions for authors, subscriptions and further details:

http://redimat.hipatiapress.com

\title{
Evaluación por Competencias en la Aplicación de un Modelo Semipresencial a las Matemáticas de titulaciones Técnicas Universitarias
}

Paz Fernández Oliveras ${ }^{1}$, María del Carmen Rodríguez Ponce $^{2}$, María Luisa Oliveras Contreras ${ }^{1}$

1) Universidad de Granada

2) Centro de Estudios Matemáticos (CEMAT), Instituto Superior Politécnico "José A. Echevarría" (CUJAE)

Date of publication: February $24^{\text {th }}, 2018$ Edition period: February 2018-June 2018

To cite this article: Fernández Oliveras, P., Rodríguez, M.C., and Oliveras Contreras, M.L. (2018). Evaluación por competencias en la aplicación de un modelo semipresencial a las matemáticas de titulaciones técnicas universitarias. REDIMAT, 7(1), 38-68. doi:

http://dx.doi.org/10.17583/redimat.2018.2191

To link this article: http://dx.doi.org/10.17583/redimat.2018.2191

\section{PLEASE SCROLL DOWN FOR ARTICLE}

The terms and conditions of use are related to the Open Journal System and to Creative Commons Attribution License (CC-BY). 


\section{Evaluación por Competencias en la Aplicación de un Modelo Semipresencial a las Matemáticas de Titulaciones Técnicas Universitarias}

$\begin{array}{lll}\text { Paz Fernández } & \text { María del Carmen } & \text { María Luisa Oliveras } \\ \text { Universidad de } & \text { Rodríguez } & \text { Universidad de } \\ \text { Granada } & \text { CEMAT, CUJAE } & \text { Granada }\end{array}$

(Received: 20 July 2016; Accepted: 06 October 2017; Published: 24 February 2018)

\section{Abstract}

Este trabajo presenta la adaptación a la modalidad semipresencial de la evaluación por competencias para las asignaturas de matemáticas de enseñanzas técnicas universitarias. Las actividades incluidas en la propuesta forman parte de los recursos para la evaluación, contribuyendo cada una de ellas a la adquisición de una o varias competencias de tipo específico, e incluso considerando su contribución a la adquisición de las competencias de tipo transversal. Se incluye el modelo de evaluación propuesto y los primeros resultados de su aplicación. Concluimos que el método de evaluación diseñado es totalmente coherente con el modelo, confiriéndole una rigurosa validez interna y su validación por expertos garantiza una alta fiabilidad de los resultados de su aplicación. Además la evaluación por competencias es muy valiosa para los profesores de Matemáticas de titulaciones técnicas.

Keywords: Evaluación por competencias, educación superior, aprendizaje semipresencial 


\section{Evaluation by Competences in a Blended Learning Model, Application to Mathematics in University Technical Degrees}

$\begin{array}{lll}\text { Paz Fernández } & \text { María del Carmen } & \text { María Luisa Oliveras } \\ \text { Universidad de } & \text { Rodríguez } & \text { Universidad de } \\ \text { Granada } & \text { CEMAT, CUJAE } & \text { Granada }\end{array}$

(Recibido: 20 Julio 2016; Aceptado: 06 Octubre 2017; Publicado: 24 Febrero 2018)

\section{Resumen}

This work presents a method of adapting to a blended learning model for the evaluation of mathematics competencies in university technical degrees. This is placed in the context of a class taught in several branches of engineering in Cuba, including an introduction specifically to the Blended Learning Model which is also designed by the authors, in which the evaluation method proposed is applied, presenting the initial results for the case under study. This approach proves completely consistent, conferring rigorous internal validity. The method of sampling students was applied and the results proved favourable. Also, as study case was examined, confirming its applicability. Its validation by experts guarantees a high degree of reliability for its future application.

Palabras clave: Competence evaluation, higher education, blended learning 
os modelos didácticos de enseñanza-aprendizaje en el sistema formativo superior europeo han experimentado cambios importantes, evolucionando desde el sistema tradicional muy dirigido y totalmente presencial, hasta modelos constructivistas que depositan en el estudiante la gestión de su aprendizaje y aceptan el trabajo fuera del entorno institucional como fundamental para dicho aprendizaje. El modelo didáctico que presenta un mayor equilibrio entre la intermediación institucional y la independencia cognitiva es el que se denomina "Semipresencial".

La modalidad semipresencial (Blended Learning), Aspden y Helm, (2004), Hourrutinier (2006), Gámiz- Sánchez, y Gallego-Arrufat, (2016), lleva años instaurada en el sistema universitario cubano, y más recientemente en las titulaciones técnicas. Sin embargo la adaptación de las metodologías didácticas a este sistema de enseñanza ha sido escasa, de forma que, generalmente, la organización de las asignaturas para impartirlas en esta modalidad ha implicado cambios muy leves en sus planteamientos. Es decir las explicaciones del profesor se han concentrado en unas pocas sesiones presenciales y el alumnado ha desarrollado los contenidos sin orientación especial, en su ámbito doméstico, en un proceso semejante al desarrollado por los alumnos que cursaban como "libres" en el sistema español, hace años. Partiendo de esta realidad y conscientes de las contradicciones que el proceso didáctico de enseñanza-aprendizaje estaba teniendo en este supuesto, se formuló, como objeto de estudio de una trabajo doctoral que involucra a las tres autoras, la adaptación al sistema semipresencial de alguna de las asignaturas de matemáticas de las enseñanzas técnicas superiores, que se imparten en las universidades de Cuba, ya que no se había realizado ninguna acción docente al respecto, contrastada mediante investigación,. Así, se ha elaborado y una propuesta didáctica completa para impartir la asignatura titulada Matemáticas III en forma semipresencial, de modo que sea aplicable en las universidades cubanas y españolas (Fernández y Oliveras, 2007). El equipo de profesoras e investigadoras que realizan esta investigación lo integran profesoras universitarias cubanas de cuya práctica profesional surgió la necesidad de un cambio metodológico en la docencia semipresencial, y por otro, profesoras universitarias españolas, expertas en matemáticas y en enseñanza superior con enfoque europeo.

De las primeras experiencias de la aplicación de las componentes organizativas de dicha propuesta, que ha estado en constante retroalimentación y mejora en los dos años académicos que lleva 
aplicándose, se ha llegado a la conclusión de que, el modelo creado está mejorando el proceso de enseñanza y los conocimientos adquiridos por los alumnos. Sin embargo, se constató que existía la necesidad de readaptar también, conforme a los objetivos de dicha propuesta, el sistema de evaluación, basándolo en la adquisición de competencias.

Así pues, en este trabajo se presenta la creación de un método de evaluación por competencias, integrado en un modelo didáctico semipresencial propuesto por estas autoras, para asignaturas de matemáticas de enseñanzas técnicas universitarias. Mostramos datos de su experimentación en una asignatura en Cuba y de su validación, como trabajo pionero, que permitirá sentar las bases para generalizar la evaluación por competencias a diversas asignaturas y titulaciones del citado país, y de cuya investigación se puedan extrapolar también aplicaciones a las universidades españolas.

Por ello, tenemos la necesidad de partir previamente de una conceptualización de lo que son las competencias profesionales en la actualidad, clave para afrontar su formación, desarrollo, y evaluación, "dando pie a nuevas estrategias y dispositivos de evaluación de competencias profesionales en el contexto de la educación superior" (Tejada y Ruiz, 2016, p. 17).

\section{Importancia de la Evaluación por Competencias}

En los nuevos planes de estudios universitarios se pretende desarrollar en los estudiantes las competencias básicas que les permitan potenciar sus conocimientos, destrezas, actitudes y valores implicados en el desempeño de sus funciones profesionales. En este sentido, los estudiantes de Ingenierías y titulaciones técnicas, precisan ser formados dentro del paradigma de la literalidad de la realidad, con una acentuada inclinación hacia la resolución de tareas operativas, que permitan trascender lo literal y lo técnico hacia pensamientos creativos (García-Restrepo, 2004).

Esta meta, que está actualmente sin alcanzar, afirma EURYDICE en su último informe temático titulado "El desarrollo de las competencias clave: desafíos y oportunidades para la política en la materia": "Europa se enfrenta a la falta de competencias en las disciplinas MCT (matemáticas, ciencias y tecnología)", (Informe EURYDICE, 2012, p. 26). La asumimos ya que nuestro enfoque entronca con el espíritu de este informe, que estudia los 
avances que se han producido en los países europeos con la incorporación de las competencias clave en sus currículos y en las prácticas docentes, desde que en el 2006 se aprobó la Recomendación del Parlamento y del Consejo Europeos sobre las competencias clave para el aprendizaje a lo largo de la vida.

En la actualidad, la mayoría de los países europeos ha reformado sus currículos para integrar las competencias transversales, [...], pero la promoción de las competencias transversales (digitales, cívicas y empresariales) se está implantando de forma muy irregular. Asimismo, en comparación con las competencias básicas, las transversales se imparten con menos frecuencia como materias independientes. Suelen integrarse en otras materias o en todo el currículo, con lo cual todos los profesores comparten la responsabilidad de enseñarlas (enfoque transversal)... (Informe EURYDICE, 2012, p. 29)

Sin embargo, el informe reconoce que, la puesta en práctica de este enfoque revela que siguen existiendo desafíos. Por ejemplo, la integración de las competencias digitales en materias como las matemáticas y las ciencias es algo sorprendentemente infrecuente en los países europeos.

Coincidimos en que:

Un enfoque transversal no solo requiere que los docentes cambien los métodos de enseñanza que tradicionalmente venían utilizando, sino que también exige un mayor grado de colaboración para desarrollar y llegar a acuerdos sobre los resultados específicos de aprendizaje que se deben alcanzar y sobre el uso de los métodos de evaluación adecuados. (Informe EURYDICE, 2012, p. 32)

En consecuencia, no podemos prescindir de una conceptualización de competencia, que fundamente nuestra evaluación:

En síntesis, el concepto de competencia es indisociable de la noción de desarrollo. No debemos olvidar que como resultante de dicho proceso de adquisición igualmente se incrementa el campo de las capacidades entrando en un bucle continuo que va desde las capacidades a las competencias y de estas a las capacidades, iniciando de nuevo el ciclo potenciador en ambas direcciones, en un continuum inagotable. (Tejada y Ruiz 2016, p. 5)

Está claro que la evaluación de las competencias, especialmente las transversales, representa un desafío en sí misma. Se necesitan para ello herramientas de evaluación que traspasen las fronteras de las materias, y 
adaptar o recrear los recursos para la realización de la evaluación de las competencias propias de cada titulación.

El contexto, por último, es clave en la definición. Si no hay más competencia que aquella que se pone en acción, la competencia no puede entenderse tampoco al margen del contexto particular donde se pone en juego. Es decir, no puede separarse de las condiciones específicas en las que se evidencia. (Tejada y Ruiz 2016, p. 5)

También es necesaria la formación al respecto, del profesorado universitario, lo que es posible en contacto con investigaciones como la que presentamos, ya que:

Las estrategias didácticas son clave; pero de la misma manera, el contenido proposicional también juega un papel fundamental; así como los ejemplos concretos. Conocer unos y otros forma parte de la formación del profesorado.” (Díez-Palomar, 2016, p. 11)

\section{Modelo Didáctico y Método de Evaluación por Competencias}

Nuestro modelo didáctico (que se expone en: Fernández et al, 2014) corresponde a los caracteres citados en Gámiz- Sánchez, y Gallego-Arrufat, (2016), obteniendo: "El efecto conjunto es explicativo de la reducción de las tasas de deserción escolar y el aumento de la nota final" en estudiantes cubanos.

Se ha creado e implementado incluyendo el método de evaluación que aquí presentamos, cuyas actividades diseñadas contribuyen a la adquisición de las competencias y además algunas se utilizan como instrumento de evaluación.

Esta concepción de la evaluación por competencias entronca con la dimensión formativa formulada por Scriven y las propuestas abordadas por Hall y Burke (2003) y Kaftan et al. (2006). Así pues

La evaluación debe de constituir una oportunidad de aprendizaje y utilizarse no para adivinar o seleccionar a quien posee ciertas competencias, sino para promoverlas en todos los estudiantes (Cano 2008, p. 44).

El diseño de evaluación por competencias y sus implicaciones han sido analizados por McDonald et al (2000), Scallon (2004), Fernández y Oliveras (2005 y 2007), Gerard (2005), De Ketele (2006) o Gerard y Bief (2008). Según Cano (2008): 
La evaluación por competencias nos obliga a utilizar una diversidad de instrumentos y a implicar a diferentes agentes. Tenemos que tomar muestras de las ejecuciones de los alumnos y utilizar la observación como estrategia de recogida de información sistemática [...]. En cualquier caso debe proporcionar información sobre la progresión en el desarrollo de la competencia y sugerir caminos de mejora. (Cano, 2008, p. 8)

El modelo didáctico que hemos elaborado está en consonancia con estas afirmaciones y comprende los siguientes elementos estructurales:

1. Plan Calendario que refleja las Secuencias de Actividades.

2. La Guía del Estudiante y del Profesor.

3. Orientaciones para el Trabajo Independiente (TI).

4. Actividades Presenciales (AP) y Actividades No Presenciales (ANP).

5. Pautas para el uso del Libro de Texto.

6. Uso de la plataforma Moodle.

El Plan Calendario planifica las Actividades Presenciales y No Presenciales (AP y ANP) y propone el tiempo promedio a dedicar por el estudiante en cada Actividad. Comprende 30 Actividades Presenciales, que representan 48 horas y 25 Actividades No Presenciales con 67 horas.

La Guía de estudio es material didáctico en formato impreso, que organiza el estudio y facilita el aprendizaje en la Modalidad Semipresencial, reforzando la actividad del profesor cuyo rol demarca.

Las orientaciones para el Trabajo Independiente TI, incluyen el desarrollo de las tareas de aprendizaje, paso a paso, los objetivos del tema y el desarrollo de orientaciones para el estudio.

Las Actividades Presenciales AP y no Presenciales ANP, incluidas en el desarrollo de cada uno de los temas, siguen la siguiente secuencia general:

- $\quad 1^{\circ}$.- AP (duración 1h): orienta el contenido del trabajo independiente del alumno.

- $\quad 2^{\circ}$.-ANP (duración y número variable según el tema)

- $\quad 3^{\circ}$.-AP (duración 2h): aclaración de dudas y realización de ejercicios

El libro de texto de cada asignatura del Plan de Estudio para las Titulaciones en Ingenierías, en Cuba se fija a nivel nacional. Se entrega a todos los estudiantes un ejemplar para ser utilizado durante el curso en condición de préstamo. En el tema: "Ecuaciones Diferenciales", el Libro de Texto es Zill (2007). 
Aprovechando las potencialidades de la Plataforma Moodle, se elaboró un recurso didáctico para ayudar al profesor a realizar el seguimiento sobre el progreso del alumno, mediante ejercicios o test de autoevaluación, trazas de los materiales de aprendizaje y la participación de los alumnos en las herramientas de comunicación.

Respecto al método de evaluación, se ha diseñado partiendo de este modelo didáctico propuesto para la enseñanza semipresencial en el caso de las Matemáticas III para ingenierías en Cuba. A partir de él y como parte del cambio metodológico, se han identificado y definido las competencias características de la citada asignatura impartida en las titulaciones de Ingeniería Informática e Ingeniería Mecánica, mostrando su contribución dentro del perfil profesional de la titulación y de su desarrollo en la sociedad cubana.

Hemos de tener en cuenta que en Europa, a raíz de la instauración del Espacio Europeo de Educación Superior (EEES), las titulaciones universitarias tienen todas sus competencias definidas, e incorporadas a los planes de estudios que las desarrollan en cada universidad, pero en Cuba esto no ocurre, por lo que la identificación de las competencias y su definición de forma explícita resulta una gran novedad en su sistema educativo universitario.

Para diseñar el sistema de evaluación, lo primero que se hizo fue: identificar las competencias específicas y transversales relacionadas con la materia "Matemáticas III", una asignatura básica para varias titulaciones de ingeniería. Una vez identificadas, éstas se pusieron en relación con el modelo didáctico, correlacionando cada competencia con la actividad o actividades de aprendizaje del modelo que contribuye a su adquisición. A partir de esto, se ha establecido el método de evaluación por competencias, adaptando la forma y modo en que las distintas actividades formativas contribuyen a la cualificación de los alumnos.

Las competencias que consideramos se adquieren, de forma total o parcial, en el Modelo Semipresencial con la citada asignatura, presentes en el tema de: Ecuaciones Diferenciales y aplicaciones, son:

Competencias Transversales (CT):

- CT1: Capacidad de autodiagnóstico del estudiante. 
- Le permite explorar su zona de desarrollo potencial, partiendo del desarrollo alcanzado, saber lo que puede hacer con otros y solicitar la ayuda requerida en el momento preciso.

- CT2: Apropiación reflexiva de los contenidos matemáticos.

- La solución de tareas por el estudiante contribuye a su aprendizaje reflexivo; es decir, a la apropiación activa de los contenidos, al desarrollo de habilidades y a la formación de valores.

- CT3: Capacidad de análisis y síntesis.

- Definidas como las capacidades para encontrar, analizar, criticar, sintetizar información de diferentes fuentes de información, así como integrar ideas y conocimientos.

- CT4: Capacidad de gestión de información.

- CT5: Capacidad de tomar decisiones, de argumentar y justificar.

- CT6: Capacidad para el aprendizaje autónomo.

- CT7: Capacidad para el uso y aplicación de las TIC.

Competencias Específicas (CE), secuenciadas en relación con los objetivos de aprendizaje:

- CE1: Identificar las Ecuaciones Diferenciales (en adelante ED).

- CE2: Clasificar las ED según el Tipo, Orden y grado.

- CE3: Obtener la solución general y/o particular de las ED de Variables Separables, Exactas y/o Lineales, de orden superior y en derivas parciales.

- CE4: Modelar problemas a través de Ecuaciones Diferenciales ordinarias (en adelante EDO) de 1er. orden y/o de orden superior, y en derivadas parciales.

- CE5: Interpretar las soluciones particulares de las EDO que modelan problemas.

- CE6: Obtener la solución general y/o particular de Ecuaciones Diferenciales de orden superior (en adelante EDOS), homogéneas y no homogéneas.

- CE7: Modelar problemas a través de EDOS.

- CE8: Hallar las soluciones de EDOS, que modelan problemas, e interpretar dichas soluciones.

- CE9: Hallar la solución de sistema de Ecuaciones Diferenciales. 
- CE10: Hallar la solución de problemas de la Física-Matemática modelados a través de Ecuaciones Diferenciales en derivadas parciales e interpretar las soluciones.

El siguiente paso en la elaboración del método fue: poner estas competencias en el contexto del modelo didáctico, enlazando a cada competencia actividades de aprendizaje, que contribuyen a su adquisición y serán instrumento de desarrollo y medida cualitativa de su logro, Figura 1.

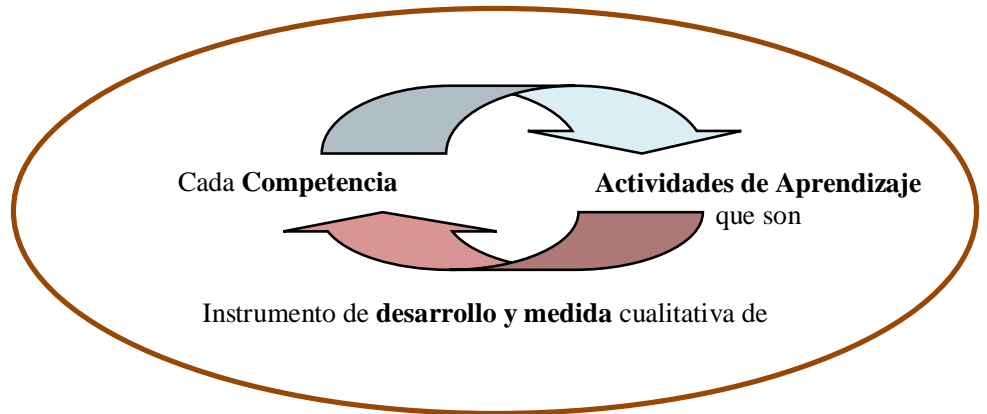

Figura 1. Competencias en el contexto del modelo didáctico propuesto

Las relaciones pormenorizadas entre cada una de las Competencias pretendidas y las Actividades de Aprendizaje se muestran en la Tabla 1. La codificación y explicación de cada una de las actividades que se recogen en la tabla es la siguiente:

- AP1: Actividad Presencial 1, de 3 horas de duración. Inicia la asignatura. Explica las características del curso y su organización. Se orientan los contenidos a estudiar de forma autónoma: definiciones básicas de E.D. y E.D. de Primer Orden.

- ANP1, ANP3: Actividades No Presenciales 1 y 3, desarrolladas de forma autónoma por los estudiantes, incluyen: orientaciones didácticas para la autogestión del conocimiento; conceptos y definiciones de E.D. en general, métodos de solución de E.D. de primer orden y de E.D. de orden superior y desarrollo de habilidades en la solución de estas ecuaciones.

- ANP2, ANP4, ANP5: Actividades No Presenciales 2, 4 y 5 con las orientaciones didácticas adecuadas. Desarrolladas de forma autónoma por los estudiantes para: aplicación de los conocimientos, 
modelar y resolver problemas, a través de la solución de ecuaciones diferenciales de primer orden, de orden superior y en derivadas parciales respectivamente.

- AP2, AP4 y AP6: Actividades Presenciales 2, 4 y 6, de 2 horas de duración cada una, cierran el tema de estudio. Momento en que los estudiantes llevan resueltos los ejercicios orientados en las actividades no presenciales que le anteceden. El profesor aclara dudas y realiza el control de desarrollo del proceso de aprendizaje, según las competencias definidas para cada caso.

Tabla 1.

Relaciones entre las competencias pretendidas y las actividades de aprendizaje

\begin{tabular}{|c|c|c|c|c|c|c|c|c|c|c|c|c|c|c|c|c|c|}
\hline \multicolumn{18}{|c|}{ Competencias. Tipo; $\mathrm{T}=$ transversal, $\mathrm{E}=$ =specífica } \\
\hline 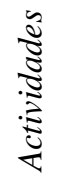 & $F$ & $\stackrel{N}{H}$ & $\stackrel{n}{\varphi}$ & 声 & 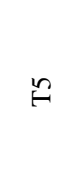 & $\stackrel{\bullet}{\varphi}$ & $\hat{F}$ & 武 & I & 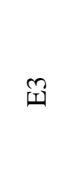 & 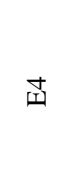 & 的 & $\begin{array}{l}0 \\
\text { III }\end{array}$ & 至 & $\underset{\infty}{\infty}$ & 至 & $\stackrel{ }{\circ}$ \\
\hline AP1 & $X$ & & & & & & & $X$ & & & & & & & & & \\
\hline ANP1 & $X$ & $X$ & $X$ & $\mathrm{X}$ & $X$ & $X$ & $X$ & $X$ & $X$ & $X$ & & & & & & & \\
\hline ANP2 & $X$ & $X$ & $X$ & $\mathrm{X}$ & $\mathrm{X}$ & $X$ & $X$ & & $X$ & $\mathrm{X}$ & $X$ & $X$ & & & & & \\
\hline AP2 & $X$ & $X$ & $X$ & $X$ & $X$ & $X$ & $X$ & $X$ & $X$ & $X$ & $X$ & $X$ & & & & & \\
\hline AP3 & $X$ & & & & & & & $X$ & & & & & & & & & \\
\hline ANP3 & $\mathrm{X}$ & $\mathrm{X}$ & $\mathrm{X}$ & $\mathrm{X}$ & $\mathrm{X}$ & $\mathrm{X}$ & $\mathrm{X}$ & $\mathrm{X}$ & $\mathrm{X}$ & $\mathrm{X}$ & & & $X$ & & & & \\
\hline ANP4 & $X$ & $X$ & $X$ & $X$ & $X$ & $X$ & $X$ & & $X$ & $X$ & $\mathrm{X}$ & $X$ & & $X$ & & $X$ & \\
\hline AP4 & $X$ & $X$ & $X$ & $X$ & $X$ & $X$ & $X$ & $X$ & $X$ & $\mathrm{X}$ & $\mathrm{X}$ & $X$ & $\mathrm{X}$ & $X$ & $X$ & $X$ & \\
\hline AP5 & $\mathrm{X}$ & & & & & & & $\mathrm{X}$ & & & & & & & & & \\
\hline ANP5 & $X$ & $X$ & $X$ & $X$ & $\mathrm{X}$ & $X$ & $X$ & $X$ & $X$ & $X$ & & $X$ & $X$ & & $X$ & & $X$ \\
\hline AP6 & $\mathrm{X}$ & $X$ & $\mathrm{X}$ & $\mathrm{X}$ & $\mathrm{X}$ & $X$ & $X$ & $X$ & $X$ & $\mathrm{X}$ & $\mathrm{X}$ & $X$ & $X$ & $\mathrm{X}$ & $\mathrm{X}$ & $\mathrm{X}$ & $\mathrm{X}$ \\
\hline
\end{tabular}

- AP3 y AP5: Actividades Presenciales 3 y 5 de 1 hora de duración cada una. En ellas se orientan los siguientes contenidos a estudiar de forma autónoma, relativos a definiciones básicas de ecuaciones diferenciales de orden superior $y$ en derivadas parciales respectivamente. 
En la Tabla 1 se muestran las relaciones de las actividades con el desarrollo de las competencias.

Se observa que las actividades AP1, AP3, AP5, son monovalentes ya que desarrollan solo las competencias E1 y T1, mientras que las AP4, AP6 desarrollan prácticamente la totalidad de las competencias. Y las actividades no presenciales desarrollan todas las competencias transversales, siendo la más polivalente la ANP5, que desarrolla además, siete competencias específicas.

\section{Procesos y Elementos que componen el método de Evaluación}

Los Elementos Didácticos que componen el método de evaluación son: el Portafolios, las Pruebas Presenciales, y los Criterios de elaboración de estos y de valoración del aprendizaje.

El Proceso de evaluación constitutivo del método comprende tres fases, conceptual y temporalmente diferenciadas: la Evaluación Frecuente (o continua), la Evaluación Parcial y la Evaluación Final.

El proceso de Evaluación Frecuente incluye: elementos del Portafolios y una Prueba Presencial corta, oral o escrita, ambos para la evaluación de las competencias desarrolladas mediante el tema tratado.

Dentro de la Evaluación Frecuente encontramos tanto elementos no presenciales (ENP) como presenciales (EP). Los ENP están incluidos en el portafolios y comprenden:

- ENP1: Evaluación del estudiante, en las tareas de aprendizaje orientadas en la Plataforma Moodle para cada tema, donde se refleja el progreso individual.

- ENP2: Presentación por escrito de preguntas sobre dudas de la teoría correspondiente al tema.

- ENP3: Presentación por escrito de esquema-resumen de aspectos teóricos esenciales del tema.

- ENP4: Recopilación de otras fuentes de información, reseña de textos consultados, indicando valoración personal del tema, con valores: 0 no adecuado o no incluye el tema, 1 adecuado, 2 bueno. 


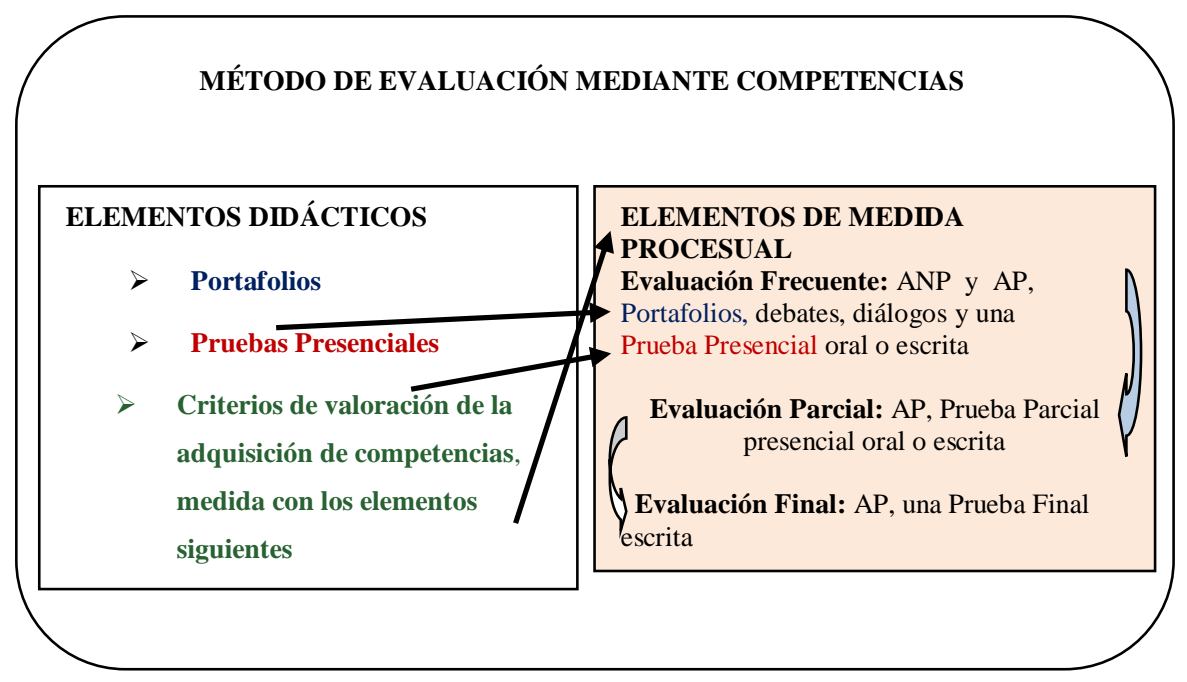

Figura 2. Elementos del método de evaluación por competencias propuesto y sus relaciones

Los EP son:

- EP1: Debate en sesiones presenciales, mediante la participación de cada estudiante en la AP de consolidación, mostrando los ejercicios a desarrollar en cada actividad ANP.

- EP2: Diálogos con el tutor. El tutor debe hacer una evaluación de cada estudiante, según comportamiento individual en el desarrollo de las ANP del tema.

- EP3: Prueba presencial corta, oral o escrita, relativa a las competencias desarrolladas en el tema, puede incluir teoría y ejercicios.

Los elementos didácticos que componen el proceso de Evaluación no Frecuente, correspondientes a las evaluaciones parciales y evaluación final, son:

- EP4: Prueba parcial presencial, que cierra el tema.

- EP5: Prueba Final escrita, donde el estudiante integre los contenidos estudiados de Ecuaciones Diferenciales y mida las competencias desarrolladas en conjunto. 
Un esquema gráfico (Figura 2) permitirá aclarar la composición del método de evaluación por competencias elaborado:

\section{Criterios para la elaboración y valoración de las pruebas de evaluación}

Las pruebas de evaluación se confeccionan teniendo en cuenta las competencias transversales y específicas para cada momento del proceso de enseñanza-aprendizaje. Al terminar la asignatura deben haberse evaluado, de una forma cualitativa y cuantitativa todas las competencias definidas previamente. Es decir el profesor tendrá una visión de las competencias logradas y su graduación, en forma pormenorizada y global.

Tabla 2.

Aporte en \% de cada una de las actividades de aprendizaje a la nota final del estudiante

\begin{tabular}{cc}
\hline Actividades de Aprendizaje & $\begin{array}{c}\text { Aporte de cada Actividad a la nota final } \\
\text { del estudiante expresado en } \%\end{array}$ \\
\hline AP1 & $5 \%$ \\
ANP1 & $10 \%$ \\
ANP2 & $10 \%$ \\
AP2 & $10 \%$ \\
AP3 & $5 \%$ \\
ANP3 & $10 \%$ \\
ANP4 & $10 \%$ \\
AP4 & $10 \%$ \\
AP5 & $10 \%$ \\
ANP5 & $10 \%$ \\
ANP6 & $10 \%$ \\
Total & $100 \%$ \\
\hline
\end{tabular}

La correspondencia entre las actividades de evaluación y las competencias se organizará en tablas, de forma particularizada para cada tema de la materia, como se verá en el ejemplo.

Para cada estudiante se tendrá la valoración de la obtención de las competencias según su comportamiento en las diferentes formas de 
evaluación, en un documento denominado hoja de desempeño (veremos una en el estudio de caso), que el profesor tendrá muy en cuenta para la Calificación Final.

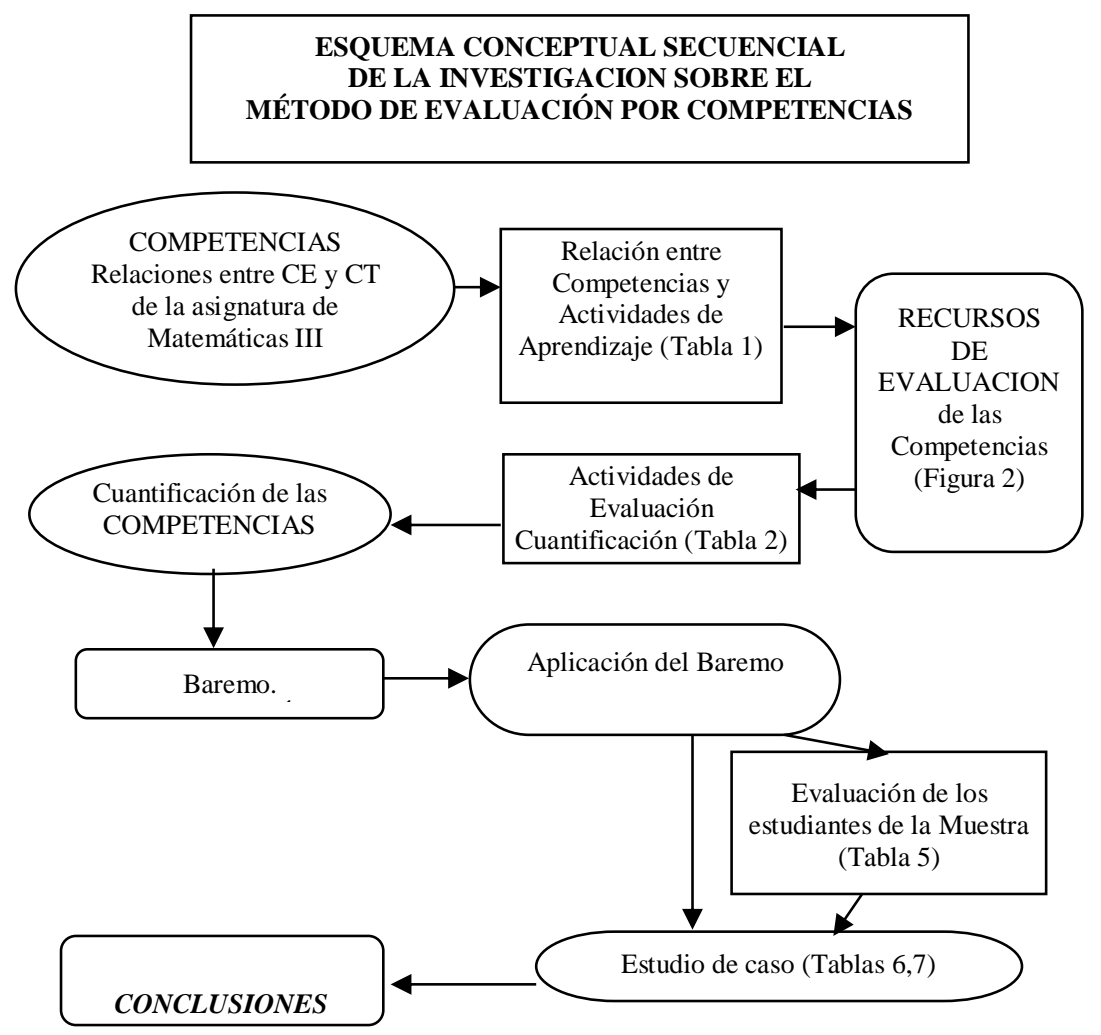

Figura 3. Síntesis de la investigación sobre el método de evaluación y su proceso secuencial

La Prueba Final (comprende ejercicios y problemas) evalúa las competencias no alcanzadas durante la Evaluación Frecuente y la Evaluación Parcial, del semestre.

La contribución porcentual de cada una de las actividades de Aprendizaje a la calificación de los alumnos se muestra en la Tabla 2. 
Especificar el porcentaje de la nota final que aporta cada una de las actividades permite: al propio estudiante conocer la importancia de las competencias implicadas en cada actividad y al profesor la cuantificación de la evaluación de las competencias, aspecto requerido por el sistema de evaluación general.

En esta metodología, todas las evaluaciones se valoran de forma cualitativa, transformándose al final cuantitativamente con los siguientes valores: 5 Sobresaliente, 4 Notable, 3 Aprobado, 2 Suspenso, para adaptarse a las pautas de evaluación generales de las titulaciones en que se ha hecho el estudio de caso.

Esta descripción del método se puede confundir con la descripción del proceso de investigación en el que se ha generado, por lo que incluimos un diagrama, aclaratorio del flujo de la investigación, en la Figura 3.

\section{Metodología de la Investigación}

El plan de esta investigación tiene dos fases, una primera teórica de elaboración del método de evaluación por competencias, y una segunda empírica de aplicación del método a un caso paradigmático. En la fase teórica han intervenido las metodologías de teorización didáctica contextualizada, con aportes de las teorías del Blended Learning (Díaz et al, 2006; Fernández y Oliveras, 2007; Gámiz- Sánchez, y Gallego-Arrufat, 2016), la teoría fundamentada, ya que el método ha emergido del proceso interpretativo llevado a cabo, (Strauss \& Corbin, 2002), el método Delphi (Oñate, et al, 1988), y la evaluación por competencias (Álvarez, 2000; Cano 2008; Delgado, 2005; Díaz et al, 2006; Gerard, 2005; Gerard \& Bief, 2008; Ketele, 2006; Tejada Fernández y Ruiz Bueno, 2016).

En la segunda fase, empírica, se ha aplicado el método a una muestra de ingenierías y de alumnos, en el entorno de las Matemáticas III, como materia común a todas las titulaciones técnicas superiores de Cuba, contexto geográfico donde se ha generado el proyecto de investigación, finalmente desarrollado con aporte investigador de España. En esta fase se ha realizado una investigación cualitativa de estudio de caso (Taylor \& Bodgan, 1986; Stake, 1999; Gurdián-Fernández, 2007), centrada en el proceso de caracterización de indicadores de adquisición de competencias y en la efectividad del método para la evaluación de dicha adquisición por los estudiantes y la consecuente valoración didáctica de los aprendizajes 
institucionales, (Scallon, 2004; Smith et al, 2003) En ambas fases se ha tratado de elaborar y validar un método de evaluación, operativo en la práctica docente universitaria, fundamentado en el rigor de su propia estructura y de su coherencia con el modelo semipresencial de enseñanzaaprendizaje establecido, ambas controladas en un proceso investigador de corte cualitativo con aportaciones cuantitativas en los aspectos puntuales que las han requerido (Eisenhart, 1988).

El proceso de elaboración teórica ha tenido, grandes aportes de las reflexiones en la propia acción, por parte las autoras que han volcado su experiencia docente en ingenierías en España y Cuba así como su capacidad de teorización didáctica, todo ello enriquecido en un proceso interactivo de elaboración cooperativa del conocimiento y su explicación.

\section{Validación del Método de Evaluación por Competencias, mediante Consulta a Expertos}

Para valorar la propuesta de evaluación por competencias se realizó una consulta a expertos antes de su implantación, empleando el método Delphi (Oñate et al, 1988). El objetivo que se persiguió fue obtener juicios críticos sobre la misma y confirmar, a partir de los resultados de esta consulta, su validez teórica.

Para corroborar que los 13 profesores seleccionados pertenecientes a universidades de Cuba y España, se consideraran como expertos se tomaron con títulos de Máster o Doctor y con más de 20 años de experiencia impartiendo matemáticas, se les aplicó una encuesta previa con el fin de valorar el coeficiente de competencia según metodología planteada por el citado método Delphi, el cual trata sobre la utilización sistemática del juicio intuitivo de un grupo de expertos para obtener un consenso de opinión .

Como resultado se obtuvo que 10 de los expertos tienen una competencia alta y 3 una competencia media. El coeficiente de competencia promedio de los expertos es alto y se utilizaron los criterios de todos ellos, tal como permite este método estadístico. Una vez aceptados todos como experto/as, se procedió a la consulta sobre nuestro método de evaluación por competencias.

El procedimiento de la consulta ha incluido actuaciones de inmersión de los expertos en el contexto: 
a) Un seminario-debate sobre la metodología de enseñanza objeto de este tipo de evaluación.

b) Una jornada-reunión de explicación pormenorizada del modelo didáctico novedoso implantado, sobre el cual se ha realizado la definición de competencias.

c) Un cuaderno en el que se explica el contenido del tema seleccionado: Ecuaciones Diferenciales, incluyendo las actividades Una encuesta en la que se preguntaba a los expertos acerca de la adecuación de las actividades de Evaluación por competencias propuestas. Es decir si las actividades eran adecuadas para medir las correspondientes competencias definidas. Se le hacían tres preguntas o ítem, además, opcionalmente, podían exponer alguna idea, crítica, recomendación, sobre la forma de evaluar.

Debían: expresar su criterio sobre la propuesta de evaluación por competencias, siguiendo las escalas de valor cualitativo: Muy Adecuada (MA); Adecuada (A); No Adecuada (MA); No Opino (NO), cuyos valores cuantitativos son: 4, 3, 2 y 1 respectivamente.

Tabla 3.

Opinión de los nueve expertos sobre la adecuación de las actividades de evaluación por competencias

\begin{tabular}{|c|c|c|c|c|c|c|c|c|}
\hline & & & Fre & encia de & punt & ción & & \\
\hline .ే & $\underset{\sum}{\mathbb{J}}$ & $b^{\circ}$ & $\underset{\ll}{\overparen{c}}$ & $b^{\circ}$ & $\begin{array}{l}\widehat{d} \\
\mathbb{z}\end{array}$ & $0^{\circ}$ & $\begin{array}{l}\widehat{\Xi} \\
0 \\
z\end{array}$ & $0^{\circ}$ \\
\hline I & 4 & 44,44 & 5 & 55,56 & 0 & 0,00 & 0 & 0,00 \\
\hline II & 4 & 44,44 & 4 & 44,44 & 0 & 0,00 & 1 & 11,11 \\
\hline III & 3 & 33,33 & 5 & 55,56 & 0 & 0,00 & 1 & 11.11 \\
\hline Total & 11 & 37,04 & 14 & 51,85 & 0 & 0,00 & 2 & 11,11 \\
\hline
\end{tabular}


Tabla 3. (.../...)

Opinión de los nueve expertos sobre la adecuación de las actividades de evaluación por competencias

\begin{tabular}{|c|c|c|c|c|c|}
\hline. & $\frac{\pi}{\overbrace{0}^{0}}$ & $\frac{\pi}{\frac{\pi}{0}}$ & $\frac{\overparen{J}}{\stackrel{\Xi}{\Xi}}$ & 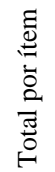 & $\begin{array}{l}\delta^{0} \\
\tilde{\theta} \\
\tilde{z} \\
\tilde{\Xi} \\
\tilde{\Xi}\end{array}$ \\
\hline I & 3,44 & 3 & 3 & 9 & 100 \\
\hline II & 3,22 & $\begin{array}{l}\text { No } \\
\text { tiene }\end{array}$ & 3 & 9 & 100 \\
\hline III & 3,01 & 3 & 3 & 9 & 100 \\
\hline Total & 3,20 & 3 & 3 & 27 & 100 \\
\hline
\end{tabular}

Los resultados estadísticos de la consulta se muestran en la Tabla 3, calculándose, para cada caso, Media, Moda y Mediana.

El análisis estadístico muestra que: todos los valores de la Media están por encima de 3, es decir entre Muy Adecuado y Adecuado, la Moda y Mediana corroboran el resultado anterior, ya que todos los valores existentes son de 3, lo que es un indicador de la validez de la propuesta a partir de los criterios dados por los expertos seleccionados. Respecto a las sugerencias solo dos expertos opinaron que solo con la práctica podrían dar ideas de posibles mejoras, que en ese momento no veían.

Por tanto consideramos validado el modelo de evaluación y listo para su implantación como parte integrante del modelo didáctico semipresencial creado, que queda así completo.

\section{Aplicación del Método de Evaluación por Competencias}

Para poner en acción en su totalidad el modelo creado, se hizo un estudio del método evaluador por competencias, en una muestra de alumnos. Mostramos resumida la operativa del método, ejemplificada para el tema 1 de la materia Matemáticas III, Explicaremos las relaciones entre tipos de competencias y 
tipos de evaluaciones, observando que hay competencias transversales y competencias específicas que se evalúan en un mismo tipo de evaluación, por ejemplo las competencias transversales 1,2 y 3 se evalúan en la evaluación no presencial 1 , que también evalúa competencias específicas. Incluimos aquí solo unos comentarios, por falta de espacio, con mayor detalle puede verse en el estudio de caso.

La codificación y explicación de las actividades de evaluación a que nos referimos es:

d) EF1: Evaluación frecuente 1, se realiza a través de la plataforma Moodle, el estudiante se autoevalúa y puede volver a someterse a evaluación de los ejercicios propuestos para esta etapa del aprendizaje, solicitando la ayuda de los elementos teóricos necesarios que brinda el programa. Mediante las actividades de esta evaluación se valoran las competencias siguientes: Las siete competencias transversales y las competencias específicas 1, 2, 3,6 у 9.

e) EF2: Evaluación frecuente 2, que se realiza a través de la plataforma Moodle, tiene mayor nivel de complejidad que el anterior. En las actividades de esta evaluación se valoran las siete competencias transversales y las competencias específicas 4, 5, 7 y 10.

f) EF3: Evaluación frecuente 3, que se realiza en la AP 2. Se desarrolla posteriormente al estudio autónomo de los estudiantes en las ANP 1 y ANP 2. Mediante las actividades de esta evaluación se valoran las competencias siguientes: Las competencias transversales: 2, 3, 5, 6 y las competencias específicas 3, 5, 8, 9 y 10.

g) ENP1: Evaluación no presencial 1, se incorpora al portafolio, el estudiante presenta por escrito el esquema-resumen de aspectos teóricos esenciales del tema. Esta evaluación proporciona información sobre el logro de todas las competencias transversales y de las específicas, todas menos las 5, 7, 8 .

h) EP1: Evaluación presencial 1 tipo parcial, se realiza al terminar el tema de Ecuaciones Diferenciales, mediante una prueba escrita que integra todos los contenidos estudiados del tema, su generalización y aplicación a otras áreas del conocimiento. 
Evalúa las competencias transversales 3, 4, 5, 6 y de las específicas, todas.

i) EP2: Evaluación presencial 2, consiste en diálogos relativos al tema con el tutor, que debe hacer una evaluación de 0 a 3 del estudiante, según su comportamiento en el desarrollo de las ANP. Todas las específicas y las transversales menos: las 4, 6, 7, son evaluadas en esta evaluación presencial, que aporta gran información sobre cada competencia y especialmente sobre la confluencia de las competencias transversales con las específicas.

j) EP3: Evaluación parcial-frecuente 3 (20\% de nota final), corresponde a las dos evaluaciones escritas cortas denominadas PE1 y PE2. Se realizan en las Actividades Presenciales 2 y 4. Permite evaluar las competencias específicas 3 a 10 y transversales: $2,3,5,6$, siendo interesante observar la confluencia entre ellas.

La evaluación cualitativa de una competencia específica alude a la/s competencia/s transversal/s relacionada/s con ella, que establece/n una matización del logro de dicha competencia, dentro del nivel cuantitativo de 2 a 5 citado.

La aplicación del baremo cuantitativo, que trata de simplificar la complejidad del proceso, establece como valor mínimo para la adquisición de una competencia el 50\% del valor que le corresponda en su valoración conjunta de todas las actividades de evaluación en las que se encuentre implicada, calculadas teniendo en cuenta la Tabla 2.

Por ejemplo, el cálculo del tanto por ciento que corresponde a cada par de preguntas escritas se realiza efectuando todas las combinaciones posibles de las cuatro calificaciones que se asignan en las dos Preguntas Escritas de la evaluación parcial 3: 2(suspenso), 3(aprobado) 4(muy bien) y 5 (excelente), (Tabla 4).

En el proceso de evaluación se integran los diferentes tipos de pruebas de evaluación siguientes y se cuantifica la aportación de cada tipo de evaluación:

a) EP4 se le ha asignado el $40 \%$ de la nota, del alumno. Se cuantifica con el tanto por ciento proporcional a las puntuaciones o Notas: 5: 40\%; 4: 32\%; 3: 24\%; 2: $0 \%$. 
b) ENP2: Evaluación No Presencial 2, presentación por escrito de preguntas redactadas sobre las dudas de la teoría correspondiente al tema: Se valora a de 0 a 2 .

c) ENP3: Evaluación No Presencial 3, presentación por escrito de esquema-resumen de los aspectos teóricos esenciales del tema: Se evalúa de 0 a 3 si entregó, o no, estos y la calidad.

d) ENP4: Evaluación No Presencial 4, recopilación de otras fuentes de información, reseña de los textos consultados, indicando su valoración personal para el tema, (con tres valores: 0 no es adecuado o no incluye el tema, 1 adecuado, 2 bueno). Se evalúa de 0 a 2 por el profesor según comportamiento del estudiante si entregó o no la consulta bibliográfica y la calidad.

e) EP5: Prueba Final escrita, donde el estudiante integre los contenidos estudiados en la asignatura.

Tabla 4.

Porcentaje de las combinaciones de calificaciones de las preguntas escritas 1 y 2 (PE1 y PE2) en la evaluación parcial 3 (EP3)

\begin{tabular}{ccc}
\hline PE1 & PE2 & $\begin{array}{c}\text { EP3: \% que representa cada } \\
\text { p.e. del 20\% asignado a este } \\
\text { tipo de evaluación }\end{array}$ \\
\hline 2 (suspenso) & 2(suspenso) & $0 \%$ \\
2 (suspenso) & 3 (aprobado) & $10 \%$ \\
2 (suspenso) & 4 (muy bien) & $12 \%$ \\
2 (suspenso) & 5 (excelente) & $14 \%$ \\
3 (aprobado) & 3 (aprobado) & $12 \%$ \\
3 (aprobado) & 4 (muy bien) & $14 \%$ \\
$3($ aprobado) & 5 (excelente) & $16 \%$ \\
$4($ muy bien) & 4 (muy bien) & $16 \%$ \\
$4($ muy bien) & $5($ excelente) & $18 \%$ \\
$5($ excelente) & $5($ excelente) & $20 \%$ \\
\hline
\end{tabular}




\section{Análisis de los Resultados Obtenidos en las Evaluaciones. Estudio de Caso}

Como muestra de la población de estudiantes a los que se aplicó el método y su evaluación se tomaron 13 alumnos de la titulación de Ingeniería Informática, cuyos datos sirvieron para ver ejemplificada la operativa del método.

No se pretende generalizar estos resultados de los estudiantes, solo ver la operatividad del método y afirmar su validez como procedimiento evaluador.

En la Tabla 5 se muestra la valoración de los alumnos de dicho grupo, mediante la aplicación del baremo a los datos de los diferentes tipos de evaluación:

Según los resultados que se muestran en la Tabla 5, se puede destacar:

a) Hay tres alumnos con valor inferior a 50\%, que correspondería a la calificación 2 y por tanto no superan la asignatura, son los codificados con: A4, A8, A10. En el intervalo entre 50 y 69\%, con una calificación de 3 , hay 4 estudiantes; y otros 4 tienen una calificación de 4 (entre 70 y 85\%), logrando solo dos el 5 por tener entre 85 y $90 \%$, estos valores de la muestra suponen unos porcentajes correspondientes a una distribución normal de los datos.

b) Respecto a la adquisición de las competencias, los valores obtenidos por los 13 alumnos tomados como muestra, nos permite afirmar:

- Con un total inferior al $50 \%$ se consideran no superadas las competencias desarrolladas con la materia, luego el $23.1 \%$ de la muestra no superan las competencias que se asignaron a la materia.

- Entre el 50 y el 70 hay $38.4 \%$ de la muestra, que han logrado de forma aceptable las competencias propias de la materia, superándola.

- Con valores superiores a 70 hay otro $38.4 \%$ de la muestra, que logran todas sus competencias específicas y desarrollan las transversales de forma excelente. 
Tabla 5.

Valoración de la muestra de alumnos en los diferentes tipos de evaluación. Asignación de su valor numérico en porcentaje.

\begin{tabular}{|c|c|c|c|c|c|c|c|c|c|}
\hline \multicolumn{10}{|c|}{ Alumno } \\
\hline \multicolumn{2}{|c|}{$\begin{array}{c}\text { Tipo de } \\
\text { evaluación }\end{array}$} & \multirow{2}{*}{$\begin{array}{l}\text { oீ } \\
\text { E }\end{array}$} & \multirow{2}{*}{$\begin{array}{l}\text { 飞 } \\
5\end{array}$} & \multirow{2}{*}{$\frac{\stackrel{2}{\gtrless}}{8}$} & \multirow{2}{*}{$\frac{\stackrel{2}{\gtrless}}{4}$} & \multirow{2}{*}{$\begin{array}{c}\stackrel{甘}{\varangle} \\
5\end{array}$} & \multirow{2}{*}{$\frac{2}{9}$} & \multirow{2}{*}{$\frac{\&}{<}$} & \multirow{2}{*}{$\frac{8}{6}$} \\
\hline Tipo 1 & EP1 & & & & & & & & \\
\hline Tipo 2 & EP2 & 3 & 2 & 1 & 1 & 2 & 3 & 3 & 4 \\
\hline Tipo 3 & EP3 & 20 & 14 & 16 & 10 & 10 & 16 & 14 & 12 \\
\hline Tipo 4 & EP4 & 40 & 32 & 24 & 24 & 0 & 32 & 40 & 32 \\
\hline Tipo 5 & ENP2 & 2 & 1 & 2 & 1 & 1 & 2 & 1 & 1 \\
\hline Tipo 6 & ENP3 & 3 & 3 & 0 & 2 & 0 & 2 & 2 & 1 \\
\hline Tipo 7 & ENP4 & 2 & 2 & 0 & 2 & 0 & 2 & 0 & 0 \\
\hline Tipo10 & $\mathrm{EF} 1$ & 10 & 5 & 8 & 6 & 6 & 8 & 8 & 6 \\
\hline Tipo11 & EF2 & 10 & 6 & 5 & 7 & 8 & 9 & 9 & 6 \\
\hline \multicolumn{2}{|c|}{ Nota final } & 100 & 70 & 64 & 57 & 34 & 82 & 87 & 68 \\
\hline
\end{tabular}

Tabla 5. (.../...)

Valoración de la muestra de alumnos en los diferentes tipos de evaluación. Asignación de su valor numérico en porcentaje.

\begin{tabular}{|c|c|c|c|c|c|c|c|}
\hline \multicolumn{8}{|c|}{ Alumno } \\
\hline \multicolumn{2}{|c|}{$\begin{array}{c}\text { Tipo de } \\
\text { evaluación }\end{array}$} & \multirow[t]{2}{*}{$\stackrel{\infty}{\infty}$} & 2 & $\stackrel{\circ}{\gtrless}$ & $\bar{Z}$ & $\frac{2}{2}$ & $\frac{m}{2}$ \\
\hline Tipo 1 & EP1 & & 9 & 5 & 5 & 5 & 8 \\
\hline Tipo 2 & EP2 & 2 & 2 & 2 & 2 & 1 & 3 \\
\hline Tipo 3 & EP3 & 10 & 18 & 10 & 12 & 14 & 16 \\
\hline Tipo 4 & EP4 & 0 & 32 & 0 & 40 & 24 & 32 \\
\hline Tipo 5 & ENP2 & 1 & 2 & 1 & 1 & 1 & 2 \\
\hline Tipo 6 & ENP3 & 0 & 3 & 0 & 0 & 2 & 3 \\
\hline Tipo 7 & ENP4 & 0 & 2 & 0 & 1 & 2 & 2 \\
\hline Tipo10 & EF1 & 5 & 9 & 4 & 6 & 8 & 10 \\
\hline Tipo11 & EF2 & 7 & 7 & 5 & 5 & 9 & 9 \\
\hline Nota fin & & 29 & 84 & 27 & 72 & 66 & 90 \\
\hline
\end{tabular}


En total los alumnos que han superado la materia en este grupo son un alto porcentaje: $76,8 \%$, de los que la mitad $(38,4 \%)$ lo hacen con excelente resultado.

En términos de competencias, según los anteriores resultados de la muestra, realizaremos un "estudio de caso" seleccionando dos estudiantes de la muestra: los de mejor y peor resultado es decir A13 y A10 respectivamente.

Tabla 6.

Valoración del logro de competencias del estudiante A10 de CE1 a CE5

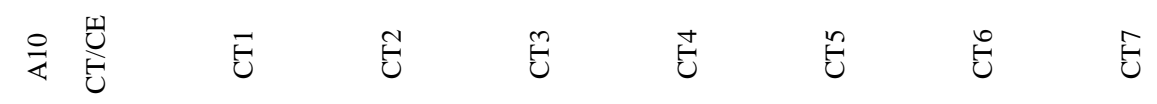

\begin{tabular}{cccccccc}
\hline CE1 & EF1=4 & EF1=4 & EF1=4 & EF1=4 & EF1=4 & EF1=4 & EF1=4 \\
& EP2=2 & ENP1=0 & EP1=5 & EP1=5 & EP1=5 & EP1=5 & ENP1=0 \\
& ENP1=0 & ENP3=0 & EP2=2 & ENP1=0 & ENP1=0 & ENP1=0 & \\
ENP4=0 & & ENP1=0 & ENP3=0 & ENP3=0 & ENP3=0 & \\
& & & ENP3=0 & ENP4=0 & ENP4=0 & ENP4=0 & \\
& & & & & \\
& Suma 6 & Suma 4 & Suma 11 & Suma9 & Suma 9 & Suma 9 & Suma 4 \\
& $<7,5$ & $<6,5$ & $<13$ NO & $<12,5$ & $<12,5$ & $<12,5$ & $<5$ NO \\
& NO & NO & & NO & NO & NO & \\
EF1=4 & EF1=4 & EF1=4 & EF1=4 & EF1=4 & EF1=4 & EF1=4 \\
EP2=2 & ENP1=0 & EP1=5 & EP1=5 & EP1=5 & EP1=5 & ENP1=0 \\
& ENP1=0 & ENP3=0 & EP2=2 & ENP1=0 & ENP1=0 & ENP1=0 & \\
ENP4=0 & & ENP1=0 & ENP3=0 & ENP3=0 & ENP3=0 & \\
& & & ENP3=0 & ENP4=0 & ENP4=0 & ENP4=0 & \\
& & & ENP4=0 & & & & \\
& Suma 6 & Suma 4 & Suma 11 & Suma 9 & Suma 9 & Suma9 & Suma 4 \\
& $<7,5$ & $<6,5$ & $<14$ NO & $<12,5$ & $<12,5$ & $<12,5$ & $<5$ NO \\
NO & NO & & NO & NO & NO & \\
\hline
\end{tabular}


Tabla 6. (.../...)

Valoración del logro de competencias del estudiante A10 de CE1 a CE5

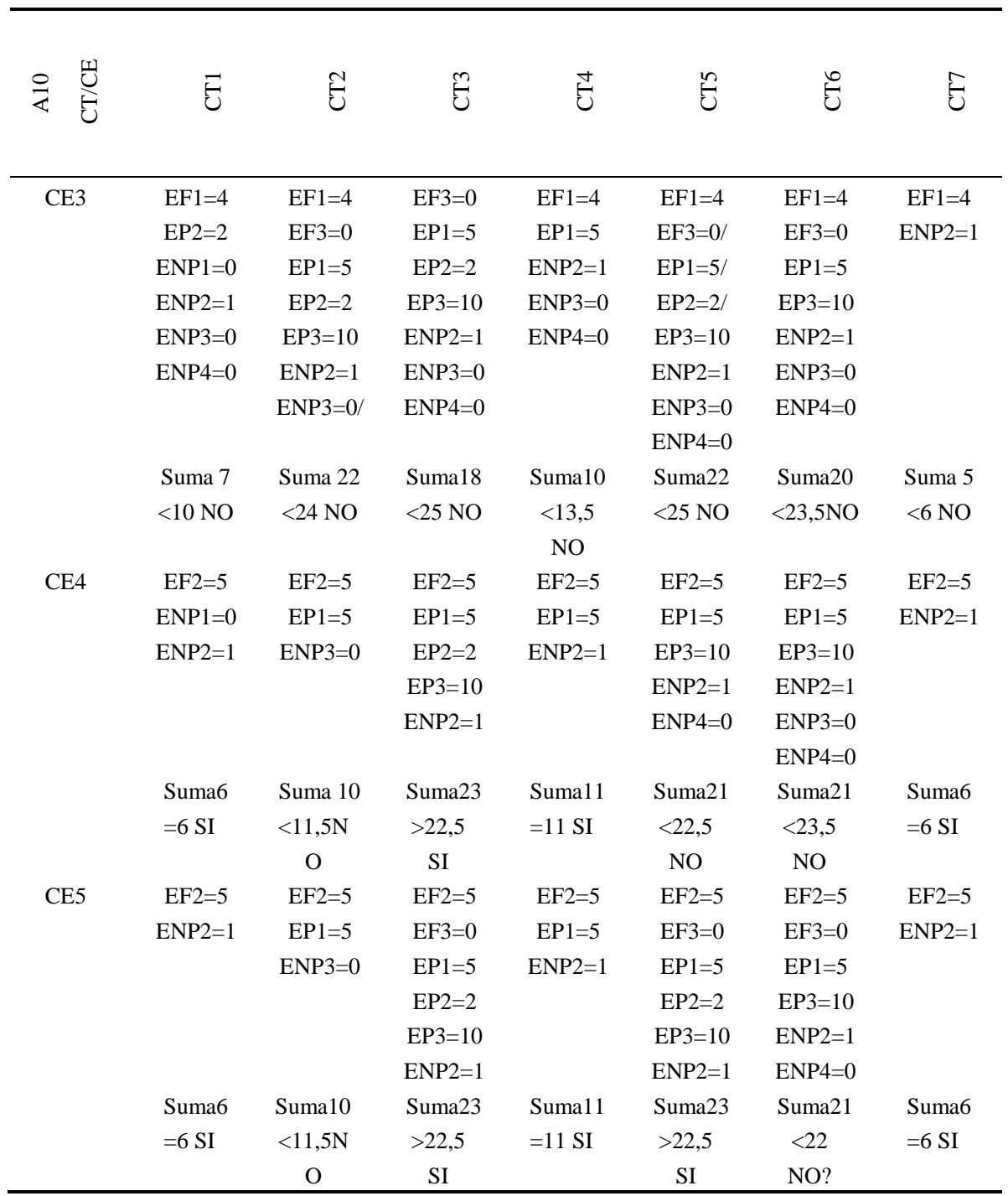


62 Fernández Oliveras et al. - Evaluación por competencias

Tabla 7.

Valoración del logro de competencias del estudiante A10 de CE6 a CE10

\begin{tabular}{|c|c|c|c|c|c|c|c|}
\hline 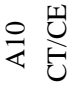 & $\bar{U}$ & 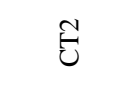 & $\stackrel{n}{0}$ & 艺 & $\stackrel{n}{v}$ & $\stackrel{\bullet}{\bullet}$ & $\hat{v}$ \\
\hline \multirow[t]{10}{*}{ CE6 } & $\mathrm{EF} 1=4$ & $\mathrm{EF} 1=4$ & $\mathrm{EF} 3=0$ & $\mathrm{EF} 1=4$ & $\mathrm{EF} 1=4$ & $\mathrm{EF} 1=4$ & $\mathrm{EF} 1=4$ \\
\hline & $\mathrm{EP} 2=2$ & $\mathrm{EF} 3=0$ & $\mathrm{EP} 1=5$ & $\mathrm{EP} 1=5$ & $\mathrm{EF} 3=0$ & $\mathrm{EF} 3=0$ & $\mathrm{ENP} 1=0$ \\
\hline & $\mathrm{ENP} 1=0$ & $\mathrm{EP} 1=5$ & $\mathrm{EP} 2=2$ & $\mathrm{ENP} 1=0$ & $\mathrm{EP} 1=5$ & $\mathrm{EP} 1=5$ & \\
\hline & & $\mathrm{EP} 2=2$ & $\mathrm{EP} 3=10$ & $\mathrm{ENP} 3=0$ & $\mathrm{EP} 3=10$ & $\mathrm{EP} 3=10$ & \\
\hline & & $\mathrm{EP} 3=10$ & $\mathrm{ENP} 1=0$ & $\mathrm{ENP} 4=0$ & $\mathrm{ENP} 1=0$ & $\mathrm{ENP} 1=0$ & \\
\hline & & $\mathrm{ENP} 1=0$ & $\mathrm{ENP} 3=0$ & & $\mathrm{ENP} 3=0$ & $\mathrm{ENP} 3=0$ & \\
\hline & & $\mathrm{ENP} 3=0$ & & & $\mathrm{ENP} 4=0$ & $\mathrm{ENP} 4=0$ & \\
\hline & Suma6 & Suma21 & Suma17 & Suma9 & Suma19 & Suma19 & Suma4 \\
\hline & $<6,5 \mathrm{NO}$ & $<23 \mathrm{NO}$ & $<18 \mathrm{NO}$ & $12,5 \mathrm{NO}$ & $<22,5 \mathrm{~N}$ & $<22,5 \mathrm{~N}$ & $<10 \mathrm{NO}$ \\
\hline & & & & & $\mathrm{O}$ & $\mathrm{O}$ & \\
\hline \multirow[t]{9}{*}{ CE7 } & $\mathrm{EF} 2=2$ & $\mathrm{EF} 2=2$ & $\mathrm{EF} 2=2$ & $\mathrm{EF} 2=2$ & $\mathrm{EF} 2=2$ & $\mathrm{EF} 2=2$ & $\mathrm{EF} 2=2$ \\
\hline & $\mathrm{EP} 2=2$ & $\mathrm{EP} 1=5$ & $\mathrm{EP} 1=5$ & $\mathrm{ENP} 2=1$ & $\mathrm{EP} 1=5$ & $\mathrm{EP} 1=5$ & $\mathrm{ENP} 2=1$ \\
\hline & $\mathrm{ENP} 2=1$ & $\mathrm{ENP} 3=0$ & $\mathrm{EP} 2=2$ & & $\mathrm{EP} 3=10$ & $\mathrm{EP} 3=10$ & \\
\hline & & & $\mathrm{EP} 3=10$ & & $\mathrm{ENP} 2=1$ & $\mathrm{ENP} 2=1$ & \\
\hline & & & $\mathrm{ENP} 2=1$ & & $\mathrm{ENP} 3=0$ & $\mathrm{ENP} 4=0$ & \\
\hline & & & & & ENP4 $=0$ & & \\
\hline & Suma5 & Suma7 & Suma20 & Suma3 & Suma18 & Suma18 & Suma3 \\
\hline & $<7,5$ & 11,5 & $<22,5$ & $<6 \mathrm{NO}$ & $>15 \mathrm{SI}$ & $<22 \mathrm{NO}$ & $<6 \mathrm{NO}$ \\
\hline & NO & $\mathrm{NO}$ & NO & & & & \\
\hline \multirow[t]{11}{*}{ CE8 } & $\mathrm{EF} 2=2$ & $\mathrm{EF} 2=2$ & $\mathrm{EF} 2=2$ & $\mathrm{EF} 2=2$ & $\mathrm{EF} 2=2$ & $\mathrm{EF} 2=2$ & $\mathrm{EF} 2=2$ \\
\hline & $\mathrm{EP} 2=2$ & $\mathrm{EF} 3=0$ & $\mathrm{EF} 3=0$ & $\mathrm{ENP} 2=1$ & $\mathrm{EF} 3=0$ & $\mathrm{EF} 3=0$ & $\mathrm{ENP} 2=1$ \\
\hline & $\mathrm{ENP} 2=1$ & $\mathrm{EP} 1=5$ & $\mathrm{EP} 1=5$ & $\mathrm{ENP} 3=0$ & $\mathrm{EP} 1=5$ & $\mathrm{EP} 1=5$ & \\
\hline & & & $\mathrm{EP} 2=2$ & & $\mathrm{EP} 2=2$ & $\mathrm{EP} 3=10$ & \\
\hline & & & $E P 3=10$ & & $\mathrm{EP} 3=10$ & $\mathrm{ENP} 2=1$ & \\
\hline & & & $\mathrm{ENP} 2=1$ & & $\mathrm{ENP} 2=1$ & & \\
\hline & & & & & $\mathrm{ENP} 3=0$ & & \\
\hline & & & & & $\mathrm{ENP} 4=0$ & & \\
\hline & Suma5 & Suma7 & Suma20 & Suma3 & Suma20 & Suma18 & Suma3 \\
\hline & $<7,5 \mathrm{NO}$ & $<10 \mathrm{NO}$ & $<22,5$ & $<7,5 \mathrm{NO}$ & $<25 \mathrm{NO}$ & $<21 \mathrm{NO}$ & $<6 \mathrm{NO}$ \\
\hline & & & $\mathrm{NO}$ & & & & \\
\hline
\end{tabular}


Tabla 7. (.../...)

Valoración del logro de competencias del estudiante A10 de CE6 a CE10

\begin{tabular}{|c|c|c|c|c|c|c|c|}
\hline$\frac{0}{\longleftarrow}$ & $E$ & $\tilde{U}$ & $\tilde{\varphi}$ & 它 & $\stackrel{n}{v}$ & ย & E \\
\hline \multirow[t]{10}{*}{ CE9 } & $\mathrm{EF} 1=4$ & $\mathrm{EF} 1=4$ & $\mathrm{EF} 3=0$ & $\mathrm{EF} 1=4$ & $\mathrm{EF} 1=4$ & $\mathrm{EF} 1=4$ & $E F 1=4$ \\
\hline & $\mathrm{EP} 2=2$ & $\mathrm{EF} 3=0$ & $\mathrm{EP} 1=5$ & $\mathrm{ENP} 2=1$ & $E F 3=0$ & $\mathrm{EF} 3=0$ & $\mathrm{ENP} 2=1$ \\
\hline & $\mathrm{ENP} 1=0$ & $\mathrm{EP} 1=5$ & $\mathrm{EP} 2=2$ & $\mathrm{ENP} 3=0$ & $\mathrm{EP} 1=5$ & $\mathrm{EP} 1=5$ & \\
\hline & & $\mathrm{EP} 2=2$ & $\mathrm{EP} 3=10$ & $\mathrm{ENP} 4=0$ & $\mathrm{EP} 2=2$ & $\mathrm{EP} 3=10$ & \\
\hline & & $\mathrm{EP} 3=10$ & $\mathrm{ENP} 1=0$ & & $\mathrm{EP} 3=10$ & $\mathrm{ENP} 2=1$ & \\
\hline & & $\mathrm{ENP} 2=1$ & $\mathrm{ENP} 3=0$ & & $\mathrm{ENP} 2=1$ & ENP3 $=0$ & \\
\hline & & ENP3 $=0$ & & & $\mathrm{ENP} 4=0$ & ENP4 $=0$ & \\
\hline & Suma6 & Suma22 & Suma17 & Suma5 & Suma22 & Suma20 & Suma5 \\
\hline & $<6,5$ & $<24 \mathrm{NO}$ & $<18 \mathrm{NO}$ & $<8,5$ & $<23,5$ & $<23,5$ & $<6 \mathrm{NO}$ \\
\hline & NO & & & NO & NO & NO & \\
\hline \multirow[t]{10}{*}{ CE10 } & $\mathrm{EF} 2=2$ & $\mathrm{EF} 2=2$ & $\mathrm{EF} 3=0$ & $\mathrm{EF} 2=2$ & $\mathrm{EF} 2=2$ & $\mathrm{EF} 2=2$ & $\mathrm{EF} 2=2$ \\
\hline & $\mathrm{EP} 2=2$ & $\mathrm{EP} 1=5$ & & $\mathrm{EF} 3=0$ & $\mathrm{EF} 3=0$ & $\mathrm{EF} 3=0$ & $\mathrm{ENP} 2=1$ \\
\hline & $\mathrm{ENP} 1=0$ & $\mathrm{EP} 4=0$ & & $\mathrm{EP} 4=0$ & $\mathrm{EP} 1=5$ & $\mathrm{EP} 1=5$ & \\
\hline & & ENP3 $=0$ & & $\mathrm{ENP} 2=1$ & $\mathrm{EP} 2=2$ & $\mathrm{EP} 4=0$ & \\
\hline & & & & $\mathrm{ENP} 3=0$ & $\mathrm{EP} 4=0$ & $\mathrm{ENP} 2=1$ & \\
\hline & & & & ENP4 $=0$ & $\mathrm{ENP} 2=1$ & & \\
\hline & & & & & $\mathrm{ENP} 3=0$ & & \\
\hline & Suma4 & Suma7 & Suma0 & Suma3 & Suma10 & Suma8 & Suma3 \\
\hline & $<7,5$ & $<31,5$ & $<22,5$ & $<23,5$ & $<35 \mathrm{NO}$ & $<33,5$ & $<6 \mathrm{NO}$ \\
\hline & NO & $\mathrm{NO}$ & NO & NO & & NO & \\
\hline
\end{tabular}

Los datos en competencias del alumno A10 se recogen en dos de sus "hojas de desempeño", que presentamos cómo Tabla 6 y Tabla 7.

Consideramos una competencia alcanzada si está superada en 4 o más de las casillas, o combinaciones de dos competencias, en las que aparece incluida en estas tablas.

Este alumno, el que tienen peores resultados, ha superado varias de las competencias aunque no las suficientes como para ser calificado apto, ya que las superadas son 10 casillas o combinaciones de dos competencias (en color blanco), de las 70 existentes. Se observa la casi superación en algunas de las 
competencias (color gris medio) y la carencia profunda en otras (color oscuro).

Se puede observar cuales son las competencias cuyas combinaciones se han superado y realizar un diagnóstico cualitativo de las competencias más avanzadas y de las menos adquiridas por el estudiante.

El análisis completo de los datos en competencias del estudiante A13, está contenido en sus "hojas de desempeño", semejantes a las anteriores y que omitimos por falta de espacio.

En ellas aparece solo una casilla no superada, la CT3-CE10, debido a la no realización de la EF3 completa, sin embargo todas las competencias han sido logradas ampliamente por este estudiante, que es el de mejores resultados.

La diferencia de 27 a 90 puntos obtenida entre los dos casos, se traduce cualitativamente en diversidad y profundidad de competencias alcanzadas.

\section{Conclusiones}

En la investigación doctoral de la que forma parte este trabajo, hemos recorrido el espectro didáctico desde la programación hasta la evaluación por competencias, pasando por la elaboración de los recursos y metodología que vehiculan el proceso de desarrollo de las citadas competencias en un modelo de tipo semipresencial.

En ese recorrido es especialmente innovador el procedimiento evaluador ya que no existe investigación sobre la evaluación por competencias dentro de la semipresencialidad aplicada a matemáticas de las titulaciones de ingenieros, en Cuba ni hemos encontrado precedente en España.

Con frecuencia se cambian los enfoques docentes en la fase de organización de la información y procesos aprendizaje, pero no en el control y evaluación de sus logros, que permanece ligado al modelo didáctico precedente, con incoherencia en el proceso de diagnóstico de los aprendizajes logrados. En este sentido se ha demostrado que el método de evaluación diseñado es totalmente coherente con el proceso previo, ya que ha utilizado las actividades del modelo de E-A, articuladas en pruebas y pautas de evaluación cualitativa y cuantitativa, lo que le confiere una rigurosa validez interna. Hemos mostrado su "validación por expertos", que le garantiza un alto grado de fiabilidad en los resultados de sus aplicaciones sucesivas. 
Se observa que incluso el alumno de peores resultados ha logrado dos competencias (CE4, CE5), tiene bastante desarrolladas otras dos (CE9, CT7), algunas en proceso de desarrollo (CE1, CE2, CE3, CE6, CE7, CT1, CT2, CT3, CT5, CT6) y otras con carencia completa, mientras que el alumno de mejores resultados las ha logrado todas, con una graduación entre ellas, que permite evaluarle en esta asignatura e incluso predecir sus mejores dotes profesionales en el futuro.

Consideramos, por todo ello, que el método presentado es un gran aporte a la investigación didáctica matemática y a la innovación en la tarea docente universitaria, especialmente en las titulaciones técnicas y que la evaluación por competencias es muy valiosa para la comunidad de profesores de Matemáticas de dichas titulaciones, colectivo que encuentra pocas investigaciones específicas en las que fundamentar con mayor rigor su práctica docente y su actualización didáctica permanente.

\section{Reconocimientos}

Agradecemos la colaboración del grupo de expertos participantes en la validación de la propuesta. Así mismo a los programas Erasmus Mundus de la Unión Europea y a la Asociación Universitaria Iberoamericana de Postgrado (AUIP), del Consejo Andaluz de Universidades y la Consejería de Innovación, Ciencia y Empresa de la Junta de Andalucía, que financiaron parcialmente la investigación.

\section{Referencias}

Álvarez, J. M. (2000). Evaluar para conocer, examinar para excluir. Madrid: Morata.

Aspden, L. and Helm, P. (2004). Making the connection in a blended learning environment. Educational Media International, 41, 3, 245 252. doi: https://doi.org/10.1080/09523980410001680851

Cano M. E. (2008). La evaluación por competencias en la educación superior profesorado. Revista de currículum y formación del profesorado, 12(3), 1-16

Díaz, M., Alfaro, I. J., Apodaca, P., Arias, J.M., García, E., Lorato, C. y Pérez, A. (2006). Modalidades de enseñanza centradas en el desarrollo de competencias. Orientaciones para promover el cambio 
66 Fernández Oliveras et al. - Evaluación por competencias

metodológico en el marco del EEES. Oviedo: Ediciones Univ. Oviedo.

Delgado, A. B. (2005). Competencias y diseño de la evaluación continua y final en el Espacio Europeo de Educación Superior. Madrid:

Dirección General de Universidades. MEC.

Díez-Palomar, J. (2016). El impacto de la investigación en la Formación de profesores. Editorial. REDIMAT, 5(3), 208-211. doi:10.4471/redimat. 2016.2342

Eisenhart, M. (1988). The ethnographic research tradition and mathematics education research. Journal for Research in Mathematics Education, 19, 99-114.

EURYDICE. (2012). El desarrollo de las competencias clave en el contexto escolar en Europa. EC-31-12-292-ES-N ISBN 978-92-9201-400-1. Brussels Press. doi:10.2797/85736.

Fernández, P. y Oliveras, M. L. (2005). Competencias docentes y recursos tecnológicos. In Congreso Internacional Virtual y Presencial sobre el Profesorado ante el Reto de las Nuevas Tecnologías en la Sociedad del Conocimiento. Granada, España.

Fernández, P. y Oliveras, M. L. (2007). Competencias Matemáticas en la Nueva Formación Científico-Tecnológica de Grado y Postgrado en el Espacio Europeo de Educación Superior. En Jornadas sobre "Investigación en el aula de Matemáticas: Competencias matemáticas". SAEM-THALES-Universidad de Granada.

Fernandez, P., Rodríguez-Ponce, M. C., Vega-Cruz, G. and Oliveras, M. L. (2014). Didactic innovative proposal for mathematic learning at the university by the blended model. Procedia - Social and Behavioral Sciences, 152, 796 - 801. doi:

https://doi.org/10.1016/j.sbspro.2014.09.323

Gámiz- Sánchez, V. y Gallego-Arrufat, M.J. (2016). Modelo de análisis de metodologías didácticas semipresenciales en Educación Superior. Educación XX1, 19(1), 39-61, doi:10.5944/ educXX1.13946

García, C. P. (2004). Más allá de la hermenéutica y la mayéutica: el pensamiento creativo. Monografía para optar al Título de Especialista en Didáctica Universitaria. Facultad de Educación, Universidad de Antioquia, Medellín, Colombia. 
Gerard, F. M. (2005). L'évaluation des compétences à travers des situations complexes. Actes du Colloque de l'Admee-Europe, IUFM Champagne-Ardenne, Reims.

Gerard, F. M. \& BIEF (2008). Évaluer des competences. Guide pratique, Bruxelles: De Boeck.

Gurdián-Fernández, A. (2007). El paradigma cualitativo en la investigación socio-educativa. San José: PrintCenter.

Hall, K. \& Burke, W. (2003). Making formative assessment work - Effective practice in the primary classroom. Maidenhead, UK: Open University Press.

Hourritunier Silva, P. (2006). La Universidad Cubana: El modelo de formación. La Habana: Félix Varela.

Kaftan, J., Buck, G., Haak, A. (2006). Using Formative Assessments to Individualize Instruction and Promote Learning. Middle School Journal, 37(4), 44-49. doi :

https://doi.org/10.1080/00940771.2006.11461545

Ketele, J. M. (2006). Caminhos para a Avaliaçao de Competencias. Revista Portuguesa de Pedagogía, 40(3), 135-147.

McDonald, R,, Boud, D., Francis, J. y Gonczi, A. (2000). Nuevas perspectivas sobre la evaluación. Boletín Cinterfor, 149, 41-72.

Oñate N., Ramos L. y Díaz A. (1988). Utilización del método Delphi en la Pronosticación: Una experiencia inicial. Cuba: Economía Planificada, 3(4), 9-48.

Rodríguez, M C., Fraga, E., Vega, G., Brito, M.L, y Fernandez Oliveras, P.(2012). Importancia del Trabajo Independiente en la enseñanzaaprendizaje de las matemáticas. Revista Pedagogía Universitaria, $17(4), 28-41$.

Scallon, G. (2004). La évaluation des apprentissages dans une approche par compétences. Québec: Ed. Du Renouveau Pédagogique.

Stake, R. E. (1999). Investigación con estudio de casos. Madrid: Ediciones Morata.

Smith, S. R., Dollase, R. H. \& Boss, J. A. (2003). Assessing Students' Performances in a Competency based Curriculum. Academic Medicine, 78(1), 97-107.

Strauss, A. \& Corbin, J. (2002). Bases de la investigación cualitativa: técnicas y procedimientos para desarrollar la teoría fundamentada. Medellín: Editorial Universidad de Antioquia. 
Taylor, S. \& Bodgan, R. (1986). Introducción a los métodos de investigación. Buenos Aires: Paidós.

Tejada Fernandez, J. y Ruiz Bueno, C. (2016). Evaluación de competencias profesionales en Educación. Superior: Retos e implicaciones. Educacion XX1, 19(1), 17-38, doi:10.5944/educXX1.12175.

Zill, D. G. (2007). Ecuaciones Diferenciales con Aplicaciones de Modelado. Madrid: Paraninfo.

Paz Fernández Oliveras es profesora contratada del Departamento de Ingeniería Civil de la Universidad de Granada, España.

María del C. Rodríguez Ponce es investigadora del Centro de Estudios Matemáticos (CEMAT), del Instituto Superior Politécnico "José Antonio Echevarría" (CUJAE), Cuba.

María Luisa Oliveras Contreras es catedrática del Departamento de Didáctica de la Matemática de la Universidad de Granada, España.

Dirección de contacto: La correspondencia directa sobre este artículo debe enviarse al autor. Dirección Postal: Universidad de Granada, ETS ICCP, Departamento de Ingeniería Civil, 18071 Granada. Email: pazferol@ugr.es 